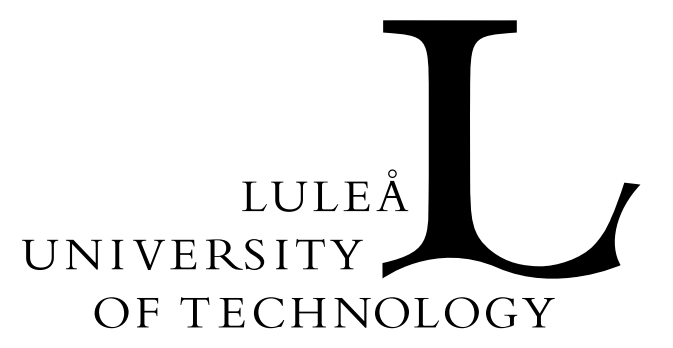

Division of Signal Processing

Visiting address: Universitetsområdet, Porsön, Luleå Postal address: SE-971 87, Luleå, Sweden

Telephone: +46920910 00. Fax: +4692072043

URL: http://www.sm.luth.se/csee/sp/

\title{
Automatic Detection of Burn-Through in GMA Welding Using a Parameteric Model
}

Stefan Adolfsson, Klas Ericsson and Anders Grennberg

In Mechanical Systems and Signal Processing, vol. 10, no. 5, pp. 633-651, 1996. 


\title{
AUTOMATIC DETECTION OF BURN-THROUGH IN GMA WELDING USING A PARAMETRIC MODEL
}

\author{
S. AdOlFsSON \\ Department of Signal Processing, University of Karlskrona/Ronneby, \\ Division of Signal Processing, Luleå University of Technology \\ K. ERICSON \\ AND \\ A. Grennberg \\ Department of Computer Science and Electrical Engineering, Division of Signal Processing, \\ Luleå University of Technology
}

(Received August 1995, accepted December 1995)

\begin{abstract}
This paper addresses the problem of automatic detection of burn-through in weld joints. Gas metal arc (GMA) welding with pulsed current is used, and welding voltage and current are recorded. As short-circuitings are common between the welding electrode and the work piece during burn-through, a short-circuit detector is developed to detect these events. To detect another specific characteristic of burn-through-a broadband long-lasting voltage component - this detector is combined with a square-law detector. This second detector is based on a non-linear modification of an autoregressive model with extra input (ARX-model) of the welding process. The results obtained from this compound detector indicate that it is possible to detect burn-through in the welds automatically. The work also indicates that it is possible to design an on-line monitoring system for robotic GMA welding.
\end{abstract}

(C) 1996 Academic Press Limited

\section{INTRODUCTION}

An ongoing process of automatisation of the production lines is implemented in industry in order to reduce production costs. Automatisation of quality control should be seen as part of the cost reduction, in addition to quality control of welding. An automatic detection system should be capable of classifying different weld defects such as porosity, metal spatter, irregular bead shape, excessive root reinforcement, incomplete penetration and burn-through.

Monitoring systems of weld parameters, such as ADM III, Arc guard, and Weldcheck have been commercially available for some years $[1,2]$. They all work in a similar way. Voltage, current and other process signals are measured, presented and compared with preset nominal values. An alarm is triggered if the difference from preset values exceeds a given threshold. However, to the authors' knowledge, the performance of ADM III, Arc guard, and Weldcheck has not been well documented.

In the field of short arc welding of steel, both physical analysis of the welding process [3-5] and statistical analysis of real welding signals have been made [6-9]. This article is based on research described in $[10,11]$ and [12]. 
The objective here is to detect burn-through automatically in pulsed-current Gas Metal Arc (GMA) welding using signal processing methods.

A brief introduction to welding technology is given in the following section. This is done in order to facilitate the understanding of the conditions for the signal processing developed in the present paper.

Section 3 describes some experiments. Burn-through is provoked in weldings in a controlled way while the weld voltage and current from this process are monitored. Some remarkable changes of the weld voltage during burn-through are observed.

As short-circuitings are common between the welding electrode and the workpiece during burn-through, a short-circuiting detector is developed in Section 4.

Due to the complexity of the physics of the welding process, a black-box model is adopted in Section 5 to further analyse if burn-through is present. The weld voltage and current during normal welding conditions are used to describe the welding process as an autoregressive model with an extra input, the current. This model is known as the ARX-model [13-15]. The least squares method is used in order to estimate the system parameters. The ARX-model, seen as a predictor, used in other situations gives prediction errors that are used in a square-law detector to detect burn-through. Short-circuitings and other welding phenomena introduce transients in the weld voltage. These transients reduce the performance of the square-law detector, which is modified using the short-circuiting detector and some transient suppressing algorithms. The two methods for detecting burn-through are combined in Section 6 and the resulting compound detector is tested on real welding signals in Section 7, giving promising results with a high rate of detection for burn-through.

\section{WELDING TECHNOLOGY}

\subsection{BURN-THROUGH}

To our knowledge there is no precise definition of burn-through in welding literature. In "AWS A3.0, Standard Welding Terms and Definitions" [16], the term 'burn-through" is described as 'a nonstandard term for excessive melt-through or a hole'. In the same standards the definition of 'excessive melt-through' is given as 'a hole through the weld metal, usually occurring in the first pass'. No definition of a hole could be found.

In the present paper, a burn-through is defined as a hole in the weld joint or in the weld metal, in which the hole can be detected by the following experiment. First, illuminate the front side of the weld joint. If any light can be detected on the rear side of the workpiece, there is a burn-through in the weld joint. An example of a burn-through can be seen in Fig. 1.

\subsection{PULSED GMA WELDING}

The GMA welding process employs a consumable wire electrode passing through a copper contact tube (Fig. 2). Electric current supports an arc flowing from the end of the electrode to the workpiece. The electrode is melted by resistive heating, and heat from the arc. The region surrounding the weld puddle is purged with shield gas to prevent oxidation and contamination of the weld joint $[5,17-19]$.

In pulsed GMA welding, the amplitude of the current alternates between two levels (Fig. 3). The advantage of this method is that the mean current, and thus the average heat input to the work piece, is lower than in direct current (DC) GMA welding. Due to the 
smaller heat transfer, it is possible to weld thinner plates with pulsed GMA than with DC GMA welding.

To limit the heat input to the workpiece, the current is low during a part of the current cycle. This part of the cycle is denoted as 'background pulse time' and represented by $T_{b}$. The current during this part of the cycle is denoted background current, and is represented by $I_{b}$. The current must exceed a critical value in order to obtain a stable arc $[17,18,20]$.

During peak pulse time, $T_{p}$, the current is high. At this stage the electrode is molten, and a droplet is detached and transferred to the workpiece. The main force for detaching a droplet and transferring it is the electromagnetic force induced by the peak current, $I_{p}$ [21].

Anode voltage fall, $U_{a n}$, column voltage fall, $U_{c o}$, and cathode voltage fall, $U_{c}$, are related by

$$
U_{a}=U_{a n}+U_{c o}+U_{c}
$$

where $U_{a}$ denotes the arc voltage [21]. $U_{w}$ denotes the weld voltage and is given by

$$
U_{w}=U_{a}+U_{e}
$$

where $U_{e}$ denotes the voltage over the wire electrode stick-out.
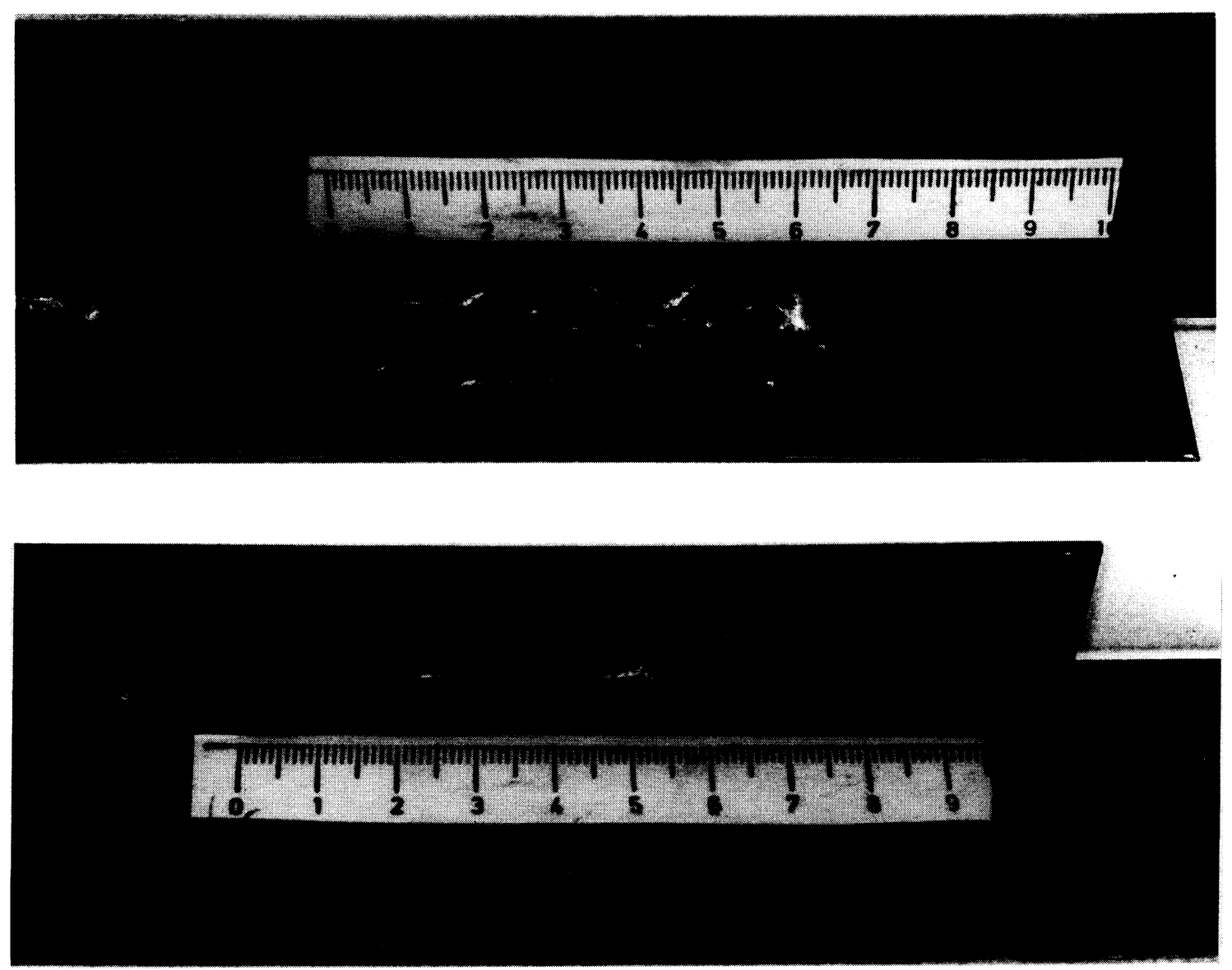

Figure 1. Phuto of (a) the front and (b) the rear side of a T-joint with burn-through. 


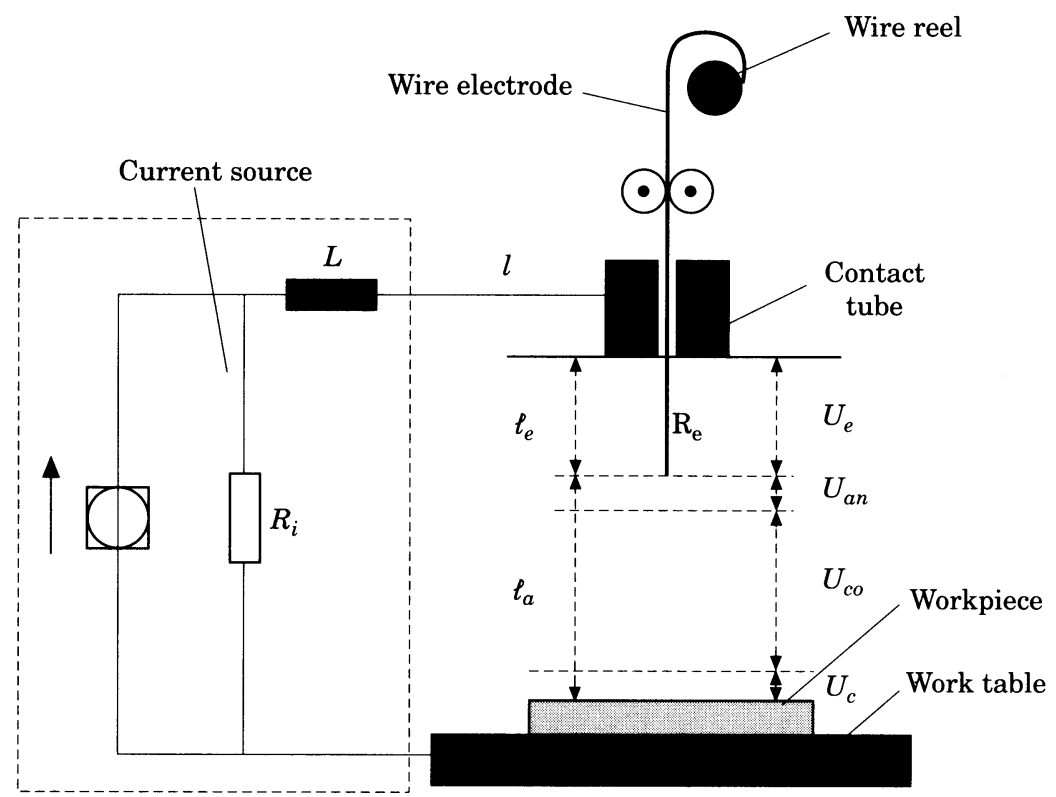

Figure 2. A schematic illustration of equipment for pulsed GMA welding. The electric current of the weld process is denoted $I$. The internal resistance of the welding source is denoted $R_{i}$. The inductance of the network is denoted $L$. The resistance of the wire electrode stick-out, i.e. the part of the electrode between the contact tube and the arc, is denoted $R_{e}$. The length of the electrode stick-out and the arc length are denoted $\ell_{e}$ and $\ell_{a}$ respectively. The voltage over the wire electrode stick-out is denoted $U_{e}$. The voltage between electrode tip and workpiece is called the arc voltage and is denoted $U_{a}$. The arc yoltage consists of three components. Anode voltage fall, $U_{a n}$, column voltage fall, $U_{c o}$, and cathode voltage fall, $U_{c}$.

The relation between the current and voltage in welding is non-linear. An experimental relation between current and arc voltage is given in [21,22] for stationary conditions.

$$
U_{a}=\beta_{1} I+\beta_{2}+\frac{\beta_{3}}{I}+\beta_{4} \ell_{a}
$$

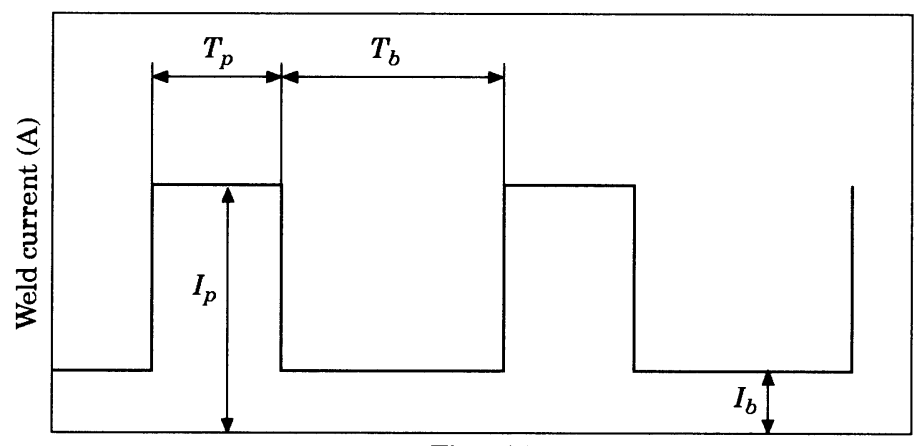

Time (s)

Figure 3. A schematic illustration of the weld current in pulsed GMA welding. $T_{p}$ and $T_{b}$ denote the peak pulse time and background pulse time respectively; and $I_{p}$ and $I_{b}$ denote the peak current and background current respectively. 


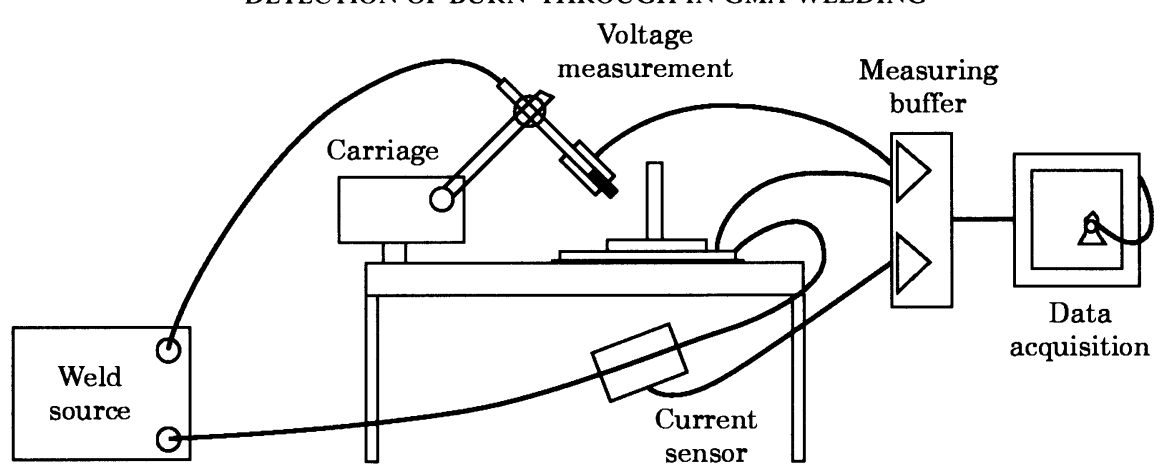

Figure 4. A schematic overview of the experimental set-up for measuring weld voltage and current from a GMA welding process. A carriage moves the torch. This is supplied with current from a weld source. The voltage between the contact tube of the weld torch and the workpiece is measured. (The second electrode is actually placed on an aluminium plate on which the work piece stands and to which the return cable of the welding source is connected. The aluminium plate is isolated from the welding table). The current fed to the torch is measured with a sensor placed around the return conductor of the weld source. The measured signals are buffered and fed to a data acquisition system.

\section{EXPERIMENTS}

\subsection{INSTRUMENTATION}

A schematic overview of the experimental set-up used in the present study is shown in Fig. 4. The experimental set-up consists of a welding source, a welding torch, a welding table and instrumentation for recording weld voltage and current. The welding torch is attached to a carriage, which runs on a rack. The carriage obtains its linear thrust from a motor mounted on the carriage. The welding torch is fixed at an angle of $45^{\circ}$ to the welding table. The distance between the contact tube tip and the plate is $15 \mathrm{~mm}$. As the torch is moved along the workpiece, the joint is filled with an alloy of electrode wire and workpiece material.

The weld voltage is measured between an electrode applied to the contact tube and a reference electrode screwed into an aluminium plate which serves as an insulated welding table. The aluminum plate is placed on an insulating plate which is placed on the welding table. The return conductor is mounted with a screw on the aluminum plate.

The purpose of this arrangement is to ensure full control of the current path [11]. The current is measured with a current sensor, LEM Module LT 500-S, equipped with a transformer. The sensor is mounted around the return conductor. Thanks to the transformer, the output is galvanically insulated from the ground. The voltage is measured with an instrumentation amplifier which is galvanically insulated from the ground by a transformer-coupled buffer.

The outputs from the current and voltage sensors are connected to a data acquisition system, a Hewlett Packard 3565S module, where the signals are filtered, sampled, digitised and temporarily stored. The sampling frequency is $32.768 \mathrm{kHz}$, and the resulting low-pass filter has an upper frequency limit of $12.8 \mathrm{kHz}$. The data are then transferred for permanent storage to a personal computer-PC 486-via an HPIB interface. The recording equipment is further specified in [11].

Commercial welding equipment, an ESAB ARISTO 500, was used for the experiment. The ESAB ARISTO 500 is able to weld with synergic control $[17,18]$. The wire feed rate was set at $5.0 \mathrm{~m} / \mathrm{min}$, which gives the parameters shown in Table 1 . The welding speed was set at $1 \mathrm{~cm} / \mathrm{s}$. The electrode filler material used in the experiment was ESAB OK 12.51 with a diameter of $1.2 \mathrm{~mm}$. The shield gas used was $90 \% \mathrm{Ar} / 5 \% \mathrm{O}_{2} / 5 \% \mathrm{CO}_{2}$. 
TABLE 1

List of nominal welding parameters

\begin{tabular}{clc}
\hline & \multicolumn{1}{c}{ Parameters } \\
\hline$I_{p}$ & peak current & $300 \mathrm{~A}$ \\
$I_{b}$ & background current & $40 \mathrm{~A}$ \\
$T_{p}$ & peak pulse time & $2.3 \mathrm{~ms}$ \\
$T_{h}$ & background pulse time & $6.2 \mathrm{~ms}$ \\
$W_{f}$ & wire feed rate & $5.0 \mathrm{~m} / \mathrm{min}$ \\
$W_{s}$ & welding speed & $10 \mathrm{~mm} / \mathrm{s}$ \\
\hline
\end{tabular}

\subsection{TEST SPECIMENS}

Burn-through was provoked using two types of specimen (Fig. 5). Part (c) shows a T-joint where gaps have been cut out in the standing plate. In the present report this specimen is called a 'T-joint with step disturbance'. Part (d) shows a T-joint where one end of the standing plate is placed on a block so that a triangular gap is achieved. This specimen is called a 'T-joint with ramp disturbance'. A third specimen shown in parts (a) and (b) is a T-joint with the standing plate in perfect contact with the laying plate. This specimen was used to produce normal or reference weldings and is thus called a 'reference T-joint'. A weld joint, judged to be an acceptable weld by an experienced welder using visual inspection, is called a 'normal weld'.

The specimens comprised of two rectangular $250 \times 50 \times 2 \mathrm{~mm}$ plates of mild steel SS1312. For the T-joint with step disturbance, the dimension of the gap was $1 \times 60 \mathrm{~mm}$ [Fig. 5(c)]. The dimension of the T-joint with ramp disturbance was $145 \mathrm{~mm}$, and $3 \mathrm{~mm}$ for the two sides of the triangle [Fig. 5(d)].

\subsection{OBSERVATION}

Two examples of recordings of the weld voltage and current are shown in Fig. 6. Parts (a) and (b) show the results of a normal weld; and parts (c) and (d) show the result of welding during burn-through. During burn-through, a broadband signal occurs in the weld voltage $[11,12]$. The signal appears to be the stochastic. The amplitude of this stochastic signal is of the order of two magnitudes less than normal peak voltage, but prolonged over several pulses. In some burn-throughs there are also several short-circuitings between the wire electrode and the workpiece. This is reflected in the weld voltage as short transients

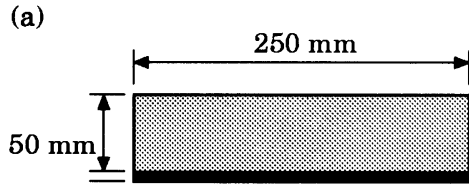

(c)

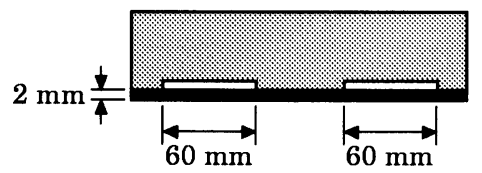

(b)

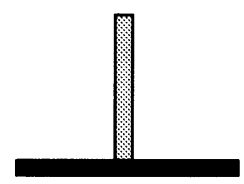

(d)

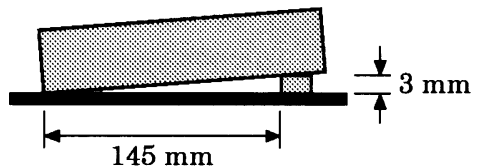

Figure 5. Illustration of steel T-joints used in the experiments to provoke burn-through in weld joints. (a) Reference T-joint, front view; (b) reference T-joint, side view; (c) T-joint with step disturbance, front view; (d) $\mathrm{T}$-joint with ramp disturbance, front view. 

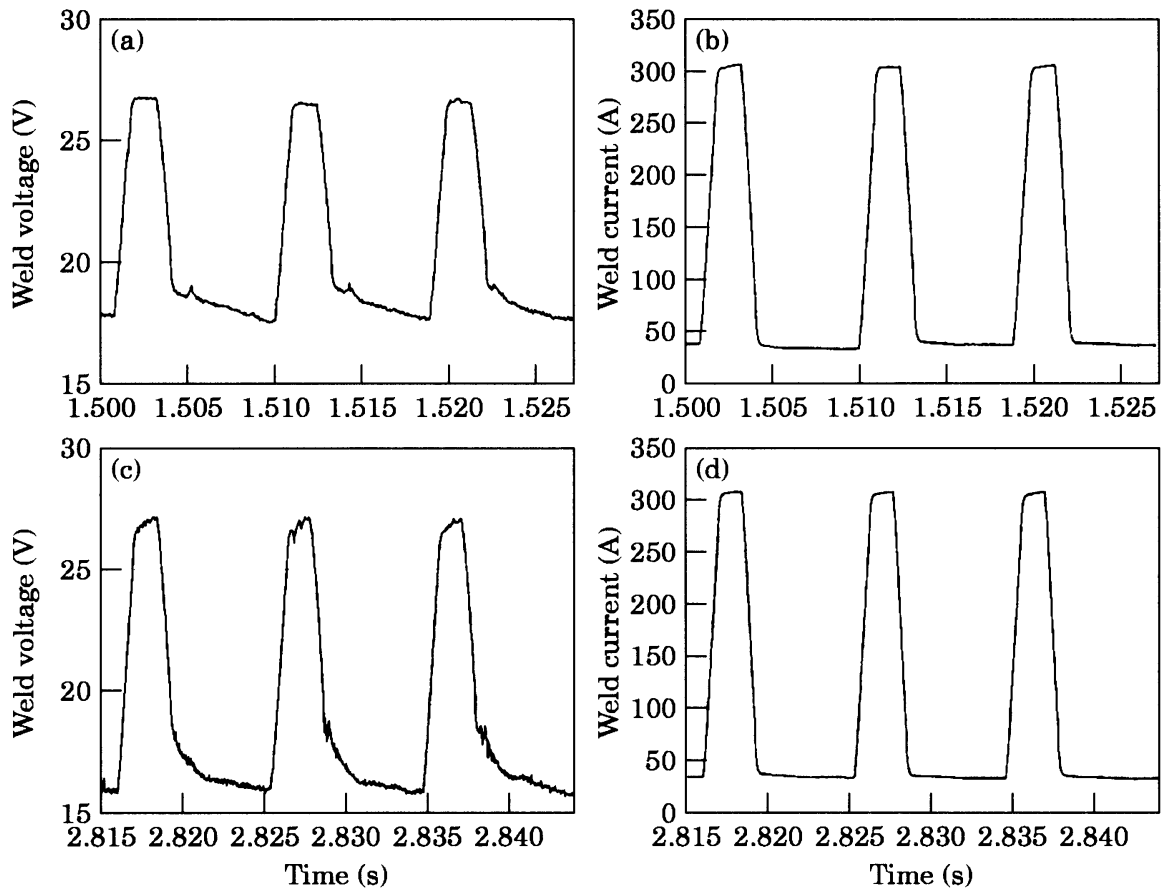

Figure 6. Diagrams of the weld voltage and current. Normal weld: (a) measured voltage and (b) measured current. During burn-through: (c) measured voltage and (d) measured current.

(in order of pulse length), almost zero voltage events. However, there are also some short circuitings in the normal welds, although they do not occur as frequently [11].

When welding a T-joint with ramp disturbance, the joint at the lower end of the specimen will be of a normal weld quality. As the welding continues, however, the weld joint deteriorates. First, there will be some burn-throughs; then the weld will deteriorate until there will be insufficient deposit to fill the joint. Thus, it can be difficult to decide whether there is a burn-through, or if it can be assumed that the weld has deteriorated completely [11]. Only the two first burn-throughs will thus be taken into account.

\section{A SHORT-CIRCUIT DETECTOR}

The purpose of the proposed detector is to test the absence contra presence of burn-through. Since consecutive large transients originating from short circuitings occur during burn-through (Section 3.3), a short-circuit detector is used.

The following detection algorithm is, therefore designed to detect short circuitings.

1. The weld voltage consists of a sequence of pulses. Give each pulse an index, $k$, defined by the position of the pulse in the sequence.

2. Short-circuit detection. Check if the actual voltage pulse number $k$ has samples less than a preset value. If this is the case, assign a parameter $S_{k}$ the value 1 , otherwise assign the value 0 . In the experiments included in the present article, the preset value is $5 \mathrm{~V}$.

3. Running mean. Calculate the running mean of the sequence $S_{k}$. Denote the output sequence $M S_{k}$. In the experiments described in a present article, the running mean 
is calculated for 11 pulses. The number of pulses is set $a d$ hoc by studying the algorithm on a few weldings.

4. Discriminator. Let the pulse sequence, $M S_{k}$, pass a level detector. If the threshold 0.5 is exceeded, an alarm is registered. The output $A M S_{k}$ is then set at 1 ; it is otherwise set at 0 .

Note that due to the running mean and the discriminator, five pulses in a sequence of 11 can indicate burn-throughs without an alarm is triggered. Five pulses correspond to a weld joint length of approximately $0.5 \mathrm{~mm}$.

\section{A SQUARE-LAW DETECTOR}

\subsection{AN ARX-MODEL}

In Section 3.3, a broadband signal component was found in the welding voltage during burn-through. To improve the detection of burn-through, the short-circuit detector developed in Section 4, is complemented with a detector that detects the broadband signal component. For that purpose a detector which uses the prediction error from an autoregressive model with extra input (ARX-model) is designed. The detector is based on the assumption that any change in weld process, which thus corresponds to a different process than the estimated ARX-process, will cause an increasing prediction error [23-25].

In order to describe the welding process statistically, it is modeled as an ARX-process with the weld current as the extra input [12-15]. An ARX-process of order $(p, r)$ is defined by the recursive relation

$$
u[n]=-\sum_{k=1}^{p} a_{k} u[n-k]+\sum_{l=0}^{r} b_{l}[[n-l]+\varepsilon[n]
$$

Here $i[n]$ is the extra input and $u[n]$ is the output. $\varepsilon[n]$ is a white noise process independent of $i[n]$. Let $u[n]=U_{w}\left(n T_{s}\right)$ and the extra input $i[n]=I\left(n T_{s}\right)$ denote the observations of the welding voltage and current at the sampling instants $n T_{s}$.

Introduce

$$
A(q)=1+\sum_{k=1}^{p} a_{k} q^{-k}
$$

and

$$
B(q)=\sum_{k=0}^{r} b_{k} q^{-k}
$$

where $q^{-1}$ denotes the delay operator and the parameters $p$ and $r$ constitute the order of the polynomials $A(q)$ and $B(q)$. Then equation (4) can be written as

$$
A[q] u[n]=B[q] i[n]+\varepsilon[n]
$$

Assuming the system to be stable and stationary, it can be written

$$
u[n]=\frac{B[q]}{A[q[} i[n]+\frac{\varepsilon[n]}{A[q]}
$$

Cf. the block scheme in Fig. 7. 
When the coefficients $a_{k}$ and $b_{l}$ are known and the input $i[n]$ is also known, it is possible to predict the output $u[n]$ by

$$
\hat{u}[n]-\sum_{k=1}^{p} a_{k} u[n-k]+\sum_{l=0}^{r} b_{l} i[n-l] .
$$

The prediction error, $\varepsilon[n]=u[n]-\hat{u}[n]$, is then a white noise process [13-15]. If it also has a known variance, $\sigma^{2}$, one could form the test quantity

$$
T q=\frac{1}{\sigma^{2}} \sum_{n=1}^{N}(u[n]-\hat{u}[n])^{2} .
$$

When the coefficients $a_{k}$ and $b_{l}$ are unknown, methods for estimating them are available [13-15]. In the present paper, the coefficients $a_{k}$ and $b_{l}$ are estimated in a least squares estimation procedure. The choice of the parameters $p$ and $r$ is made according to the Akaike Information Criterion (AIC). This procedure also gives an estimate $\hat{\sigma}^{2}$ of the variance $\sigma^{2}$ of the prediction error. These estimated values are used to calculate a prediction $\hat{u}^{\prime}[n]$ with the corresponding prediction error $e[n]=\left(u[n]-\hat{u}^{\prime}[n]\right)$. Thus, one can obtain the test quantity

$$
T Q=\frac{1}{\hat{\sigma}^{2}} \sum_{n=1}^{N}\left(u[n]-\hat{u}^{\prime}[n]\right)^{2} .
$$

If the estimation of the $\mathrm{ARX}$-model is correct the prediction error, $e[n]$, is white.

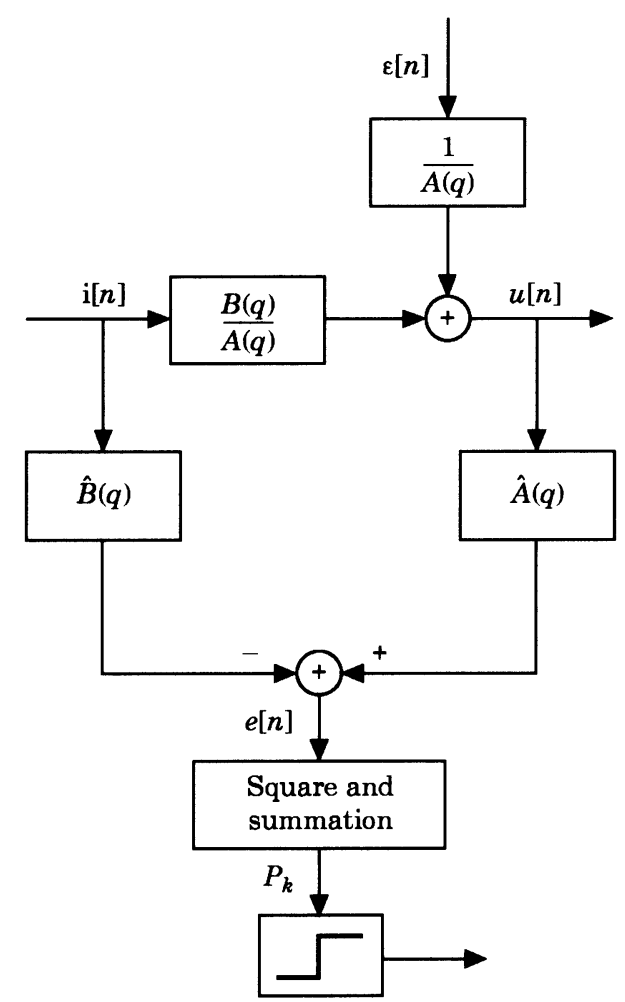

Figure 7. Block diagram of the square-law detector. 
The following preliminary square-law detector is proposed.

1. Subtract the mean values from the weld voltage and current before they are filtered by the estimate ARX-model [15].

2. The weld current consists of a sequence of pulses. Give each pulse an index $k$, defined by the position of the pulse in this sequence.

3. Locate the start sample of the first current pulse. Then locate the corresponding sample of the prediction error. Calculate the sum of the squares of $N$ consecutive samples of the prediction error as:

$$
P_{k}=\frac{1}{N \hat{\sigma}^{2}} \sum_{i=1}^{N} e^{2}[i]
$$

where $\hat{\sigma}^{2}$ is the estimated variance of the noise $\varepsilon[n]$ from the ARX process in equation (4).

4. Repeat step 3 for each succeeding current pulse.

5. Form the test quantity $T Q=N \cdot P_{k}$. If this is found to be greater than a specified threshold $G$, the burn-through indicator turns on

If the prediction error is a white gaussian process the test quantity $T q$ is $\chi^{2}$ disributed with $\mathbf{N}$ degrees of freedom. One can then select significance level and calculate a threshold $G$ using the known generic density function of the $\chi^{2}$ distribution.

If the ARX-model is not properly estimated, the prediction error, $e[n]$, will probably not be Gaussian, nor will it be a white process. The distribution of the test quantity $T Q$ will not be $\chi^{2}$ distributed, not will it have a known generic density function. It must be determined by experiment.

However, if the deviations are moderate $T Q$ can be assumed almost $\chi^{2}$-distributed.

\subsection{ESTIMATION OF THE ARX-PROCESS}

The parameters of the ARX-process were identified from the weld voltage and current of a normal weld. Before estimating the ARX-process, the mean was subtracted from the weld voltage and current [15]. The number of data points was taken as $M=4000$, i.e. the data accomplished approximately 14 current pulses. An eighth-order ARX-model was found to minimise the Akaike Information Criterion [12,14,15]. The results of the estimation of an eighth-order ARX-model are given in Table 2. An example of a sequence of the prediction error during a normal weld is shown in Fig. 8.

$\chi_{N}^{2}$-tests were applied to the prediction error in order to test if the prediction error was Gaussian or Laplacian distributed [26]. The outcome of these tests-not included in this article-shows that the hypothesis, $e[n]$ is Gaussan or Laplacian distributed, could be rejected at the significance level of $\alpha=0.001$. No other known generic distribution that fit the stochastic variable $P_{k}$ was found by the authors.

\subsection{EVALUATION OF THE SQUARE-LAW DETECTOR}

In order to evaluate the square-law detector, two batches, each of 400 samples of the parameter $P_{k}$ originating from data from normal welds and welds during burn-through respectively, are used. These are illustrated in Fig. 9. A sample length of 400 pulses corresponds to a 4-cm weld joint. A corresponding relative frequency plot is shown in Fig. 10 (a) and (e). High-voltage transients are represented as a bar at the value 6 . This bin represents the relative frequency for all $P_{k}: s$ values above 6 in the diagrams in Fig. 10 (a) and (e). The figure shows that the two relative frequency distributions are not well separated. Thus, this detector is unsuitable for detecting burn-through. 
TABLE 2

Parameter estimates with estimated standard deviations for the eighth-order ARX-process of the prediction error. The number of data points was $M=4000$

\begin{tabular}{|c|c|c|c|c|c|}
\hline \multicolumn{5}{|c|}{ ARX Model } & \\
\hline $\begin{array}{l}\text { Parameter } \\
\text { Estimated parameter } \\
\text { Standard deviation }\end{array}$ & $\begin{array}{c}a_{1} \\
-1.0987 \\
\pm 0.0112\end{array}$ & $\begin{array}{r}a_{2} \\
0.3833 \\
\pm 0.0166\end{array}$ & $\begin{array}{c}a_{3} \\
-0.4637 \\
\pm 0.0171\end{array}$ & $\begin{array}{c}a_{4} \\
0.1704 \\
\pm 0.0179\end{array}$ & \\
\hline $\begin{array}{l}\text { Parameter } \\
\text { Estimated parameter } \\
\text { Standard deviation }\end{array}$ & $\begin{array}{c}a_{5} \\
-0.0902 \\
\pm 0.0178\end{array}$ & $\begin{array}{c}a_{6} \\
0.0904 \\
\pm 0.0171\end{array}$ & $\begin{array}{c}a_{7} \\
-0.0639 \\
\pm 0.0164\end{array}$ & $\begin{array}{c}a_{8} \\
0.0825 \\
\pm 0.0108\end{array}$ & \\
\hline $\begin{array}{l}\text { Parameter } \\
\text { Estimated parameter } \\
\text { Standard deviation }\end{array}$ & $\begin{array}{r}b_{0} \\
0.0426 \\
\pm 0.0005\end{array}$ & $\begin{array}{c}b_{1} \\
-0.0565 \\
\pm 0.0010\end{array}$ & $\begin{array}{c}b_{2} \\
0.0272 \\
\pm 0.0013\end{array}$ & $\begin{array}{c}b_{3} \\
-0.0256 \\
\pm 0.0015\end{array}$ & \\
\hline $\begin{array}{l}\text { Parameter } \\
\text { Estimated parameter } \\
\text { Standard deviation }\end{array}$ & $\begin{array}{r}b_{4} \\
0.0145 \\
\pm 0.0016\end{array}$ & $\begin{array}{c}b_{5} \\
-0.0064 \\
\pm 0.0015\end{array}$ & $\begin{array}{c}b_{6} \\
0.0058 \\
\pm 0.0013\end{array}$ & $\begin{array}{c}b_{7} \\
-0.0048 \\
\pm 0.0012\end{array}$ & $\begin{array}{c}b_{8} \\
0.0036 \\
\pm 0.0006\end{array}$ \\
\hline $\begin{array}{l}\text { Parameter } \\
\text { Estimated parameter }\end{array}$ & $\begin{array}{c}\sigma^{2} \\
0.0035\end{array}$ & & & & \\
\hline
\end{tabular}

\subsection{DEVELOPMENT OF A MODIFIED SQUARE-LAW DETECTOR}

When estimating the ARX-process it was found that the prediction error sequence for a normal welding is non-Gaussian. The developed detector in Section 5.1 could still be used but with difficulties in calculating the detection threshold as it was not possible for the authors to express the relative frequency in known generic distribution functions. It is, however, possible to find orders and coefficients such that the prediction error sequence passes several whiteness tests $[15,26]$.

Figure 8 indicates that the error sequence has periodically occurring spikes. This is due to the welding process being non-linear [equation (3)] and that it is still modeled with a linear model. Using a linear model is yet suitable because most of the time the current takes one of two values. When the current and voltage suddenly changes the model cannot follow the non-linear welding process which is seen from the spikes. The remedy chosen

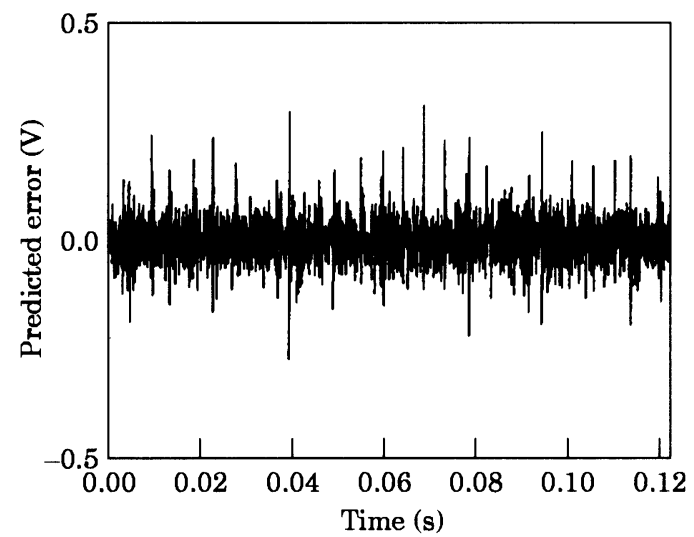

Figure 8. The prediction error of the estimated ARX-model during normal welding. The spikes appear mainly where the weld current changes. 

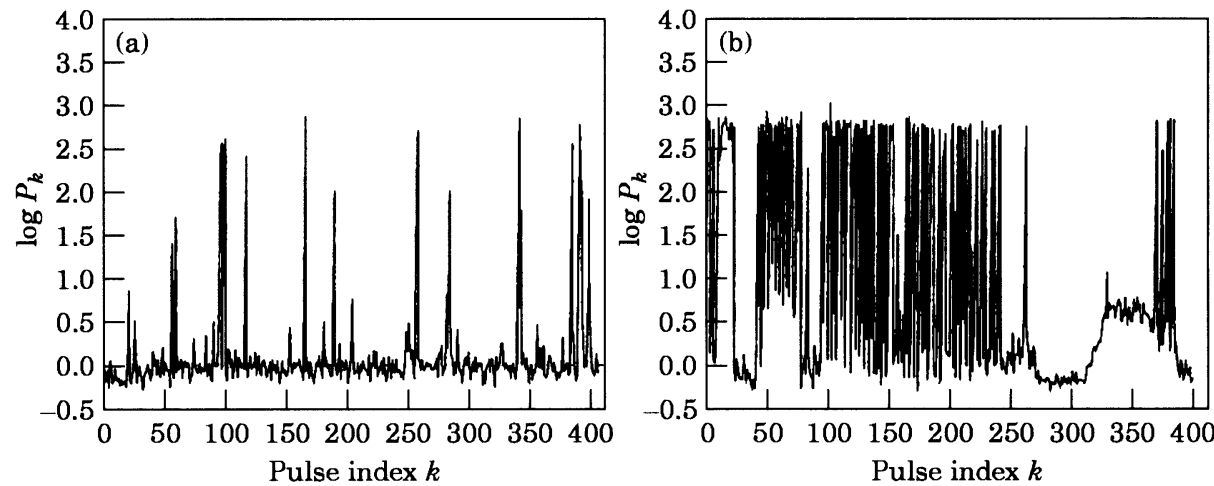

Figure 9. The parameter $P_{k}$ during normal weld. (b) The parameter $P_{k}$ during burn-through. $P_{k}$ is based on 278 samples of the prediction error. Note the logarithmic scale.

is to use a limiter on the amplitude of the prediction errors before using it in the square-law detector.

The value of the limiter is set $a d$ hoc at twice the standard deviation of the prediction error during burn-through without short-circuiting. This is empirically found to be $0.1 \mathrm{~V}$ in the test set in the present article.

As mentioned before, Fig. 10 (a) and (e) show the relative frequency plots of $P_{k}$ for a normal weld, and a weld during burn-through respectively. The influence of the limiter on the relative frequency plot of the prediction error is seen in Fig. 10 (b) and (f). It can be

(a)

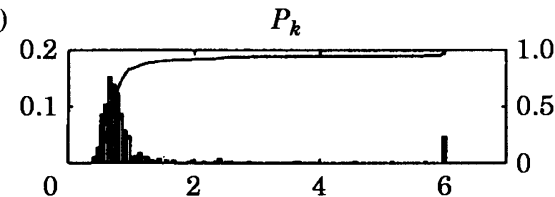

(b)

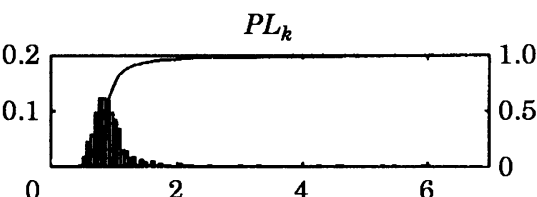

(c)

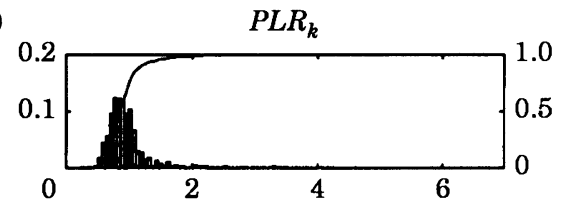

(d)

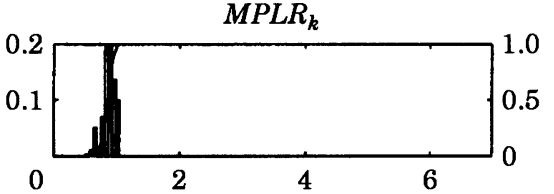

(e)

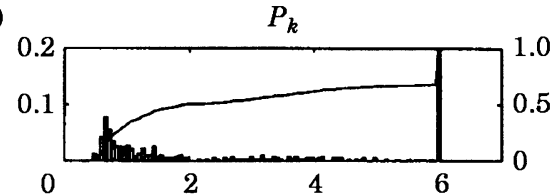

(f)

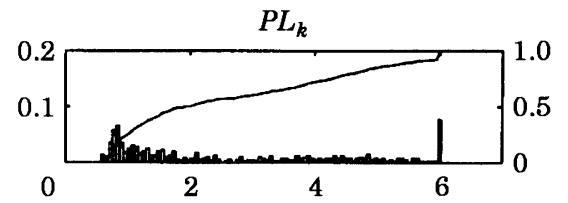

(g)

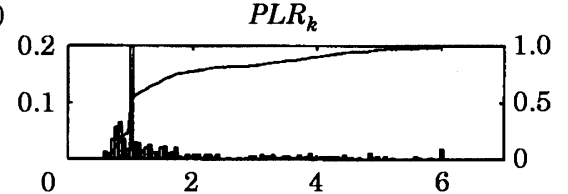

(h)

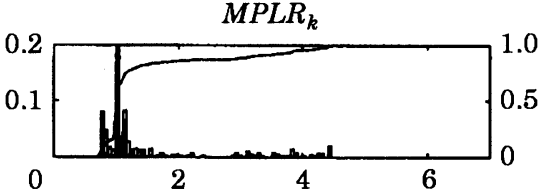

Figure 10. Illustration of the influence of the improvements of the detection algorithm using relative frequency and cumulative relative frequency plots (see the left part of Fig. 11). The left ordinate of the diagrams denotes the relative frequency and the right ordinate, the cumulative relative frequency. Parts (a) and (e) show relative frequency plots for a normal weld and a weld during burn-through, respectively, when the detector developed in Section 5.1 is used. Parts (b) and ( $f$ ) show the corresponding plots when the prediction errors are limited. Parts (c) and (g) show the plots after removal of samples originating from short-circuiting sequences, and the corresponding parameter $P L R_{k}$ is set to 1 . Parts (d) and (h) show the plots after the median filtering has been included in the process. 
seen that the relative frequency bin at 6 has been reduced for both a normal weld, and a burn-through.

As mentioned in Section 3.2, short circuitings occur frequently even if there is no burn-through in the weld. To reduce the influence from the short circuitings on the squarelaw detector the value of the detector $P L$ is set to 1 for short-circuited pulses. That is, it is assumed that the pulse originates from a normal weld. The outcome of this filter procedure is denoted $P L R_{k}$. Detection of short circuitings are made with the detector described in Section 4.

The influence of the removal of the short circuitings on the relative frequency plot of the limited prediction error is seen in Fig. 10 (c) and (g). It is seen that the variance is further reduced.

Finally, median filtering is used to enhance the difference between normal welds and welds with burn-through. The length of the median filter is chosen to be 21 . The influence of the filtering can be seen in Fig. 10 (d) and (h).

A modified square-law detector that is compensated for the non-linearities of the process can thus be designed as follows.

1. Subtract the mean values from the weld voltage and current before they are filtered by the estimated ARX-model.

2. Limiter. Apply a two-sided limiter to the prediction error in order to reduce the effect of the extreme values. The value of the limiter is set at twice the standard deviation of the prediction error during a burn-through without short circuitings. This is empirically found to be $0.1 \mathrm{~V}$ in the test set in the present article. The output sequence from the limiter can be written:

$$
f[i]=\mathrm{L}[\mathrm{e}[\mathrm{i}]], \quad i=1, \ldots, N .
$$

3. The weld current consists of a sequence of pulses. Give each pulse an index, $k$, defined by the position of the pulse in this sequence.

4. Locate the start sample of the first current pulse. Then locate the corresponding sample of the amplitude limited prediction error. Calculate the sum of the squares of $N$ consecutive samples of the prediction error $P L_{k}$ ( $L$ stands for limited) as:

$$
P L_{k}=\frac{1}{N s^{2}} \sum_{i=1}^{N} f^{2}[i],
$$

where $s^{2}$ is the estimated variance of the amplitude limited prediction error from the sequence used when identifying the ARX model. In the experiment described in the present article, the duration of a pulse period of the current is approximately 278 samples. Thus $N$ is set at 278 .

5. Short-circuit detection. Check if the actual voltage pulse has samples of less than a specified value. If so, assign the corresponding $P L_{k}$ the value 1 , i.e. assume that the voltage pulse originates from a normal weld. If this is not the case, retain the calculated value from step 4 . Denote the new sequence $P L R_{k}(R=$ remove short circuitings).

6. Repeat step 4 and 5 for each succeeding current pulse.

7. Median filtering. Apply a median filter to the sequence $P L R_{k}$. Call the resulting sequence $M P L R_{k}$. In the experiment outlined in the present article, the length of the median filter is chosen to be 21. The influence of the filtering can be seen in Fig. 10 (d) and (h). The length of the median filter is set ad hoc by studying the performance of the algorithm on a few weldings. 
8. Discriminator. Let the pulse sequence, $M P L R_{k}$, pass a level detector. If a specified threshold is exceeded, an alarm is registered. The output $D_{k}$ is then set at 1 ; otherwise it is set at 0 .

In the experiment of the present report the threshold is set ad hoc. It can be seen from Fig. 10 (d) and (h) that the plots of the relative frequencies of the quadratic sums still overlap. It was not possible for the authors' to express the relative frequency in known generic distribution functions. It seems, however, possible to find a reasonable detection limit to discriminate between the two cases. The level is set $a d$ hoc at 2.0 in the present article.

Note that due to the median filtering, 10 pulses in a sequence of 21 can have values that exceed the threshold without the alarm being set. Ten pulses correspond to a weld joint length of approximately $1 \mathrm{~mm}$.

\section{A COMPOUND BURN-THROUGH DETECTOR}

\subsection{SYNTHESIS}

There are now two possibilities to indicate burn-through: with the modified square-law detector, and with the short-circuit detector. Only one of these needs to be set in order to indicate a burn-through. Thus, a compound detection algorithm is composed by connecting the two detectors in parallel. This is illustrated by the block scheme in Fig. 11.

\subsection{TEST OF THE COMPOUND DETECTOR}

The compound detector was tested on 33 specimens. Eleven experiments were conducted for the T-joint with step disturbance, reference T-joint and ramp disturbance per se. The recording time of the measured signals was 4,5 and $10 \mathrm{~s}$ for step disturbance, reference and ramp disturbance respectively. There were 34 burn-throughs in all in the weld joints produced, 11 burn-throughs in the T-joint with step disturbance, 23 burn-throughs in the $\mathrm{T}$-joint with ramp disturbance, and no burn-throughs in the reference $\mathrm{T}$-joints.

The test was designed as follows: if the alarm turns on and there is a burn-through, the test results in a detection; and if the alarm does not turn on, there is no detection. If the alarm turns on and there is no burn-through, the test results in a false alarm. As mentioned in Section 5.4, the threshold was set at 2.

\section{RESULTS}

The results of the test are shown in Table 3. To illustrate the results of the data, the test result for a T-joint with a step disturbance, is shown in Fig. 12. (cf. with Fig. 11). The top diagram of the figure, part (a), shows the weld voltage, and part (b) shows the $P L_{k}$. Part (c) shows the corresponding $S_{k}, A M S_{k}, D_{k}$ parameters and Alarm. Part (d) of the figure shows the actual location of the burn-throughs. Part (e) shows the type and position of the disturbance along the weld joint.

\section{DISCUSSION}

Due to the complexity of the physics of the welding, a black-box model is adopted to check if burn-through is present. Both the ARX-model parameters, estimated from measured weld voltage and current, and the prediction error, are easy to obtain. The suggested detection method thus requires little a priori knowledge of the weld process. The algorithm does not require a large storage capacity or complicated calculations. Then 


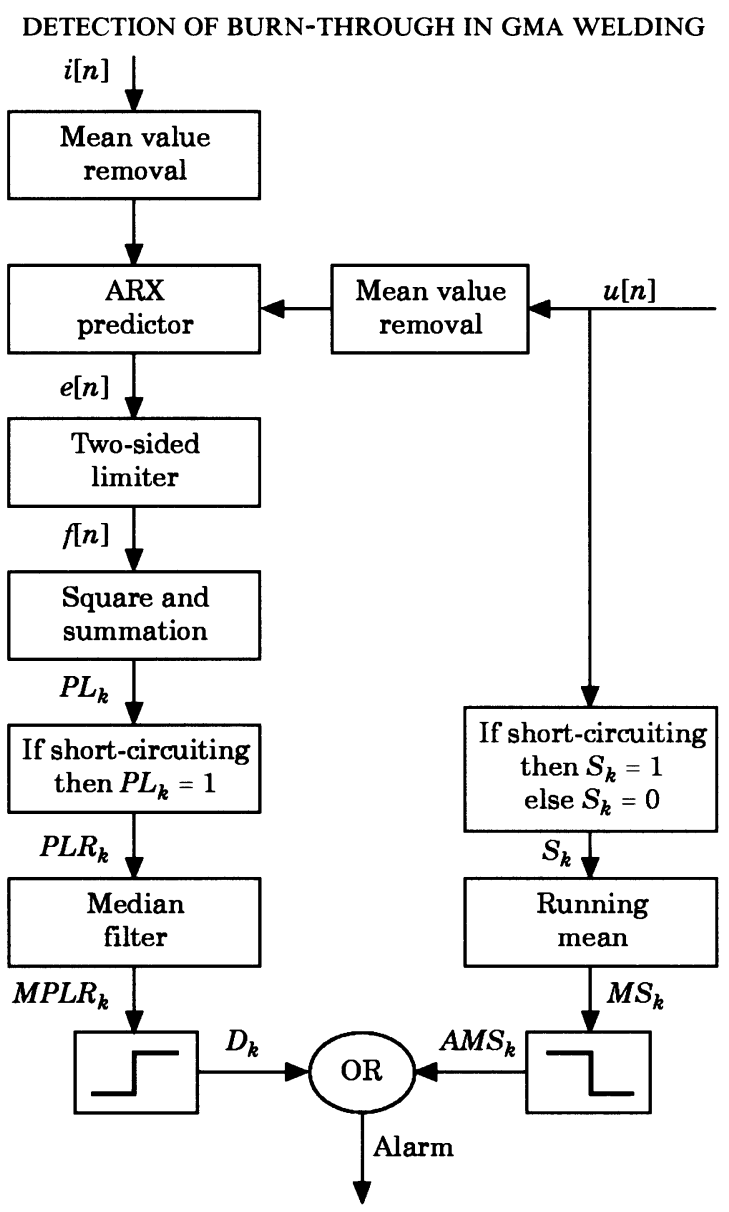

Figure 11. Block diagram of the compound detector.

the algorithm can be implemented on a digital signal processor and fast signal processing can be achieved. Thereby it is possible to build an on-line automatic detection system for detection of burn-through in robotic GMA welding.

Note that neither the short circuitings nor the broadband signal component alone is enough to detect burn-through [Fig. 12 (c) and (d)]. This exemplifies the need of the more complex compound detector.

Although the prediction error will no longer be white, preliminary comparative calculations have shown that the number of system parameters in the ARX-model can be reduced further without affecting the test results negatively (not described in this article).

TABLE 3

The results of the test of the compound detector (the threshold for the alarm is set at 2)

\begin{tabular}{lcccc}
\hline \multicolumn{1}{c}{ Type of T-joint } & Number of burn-through & Detection & Non-detection & False alarms \\
\hline Reference & 0 & - & - & 0 \\
Step disturbance & 11 & 11 & 0 & 1 \\
Ramp disturbance & 23 & 20 & 3 & 1 \\
\hline
\end{tabular}



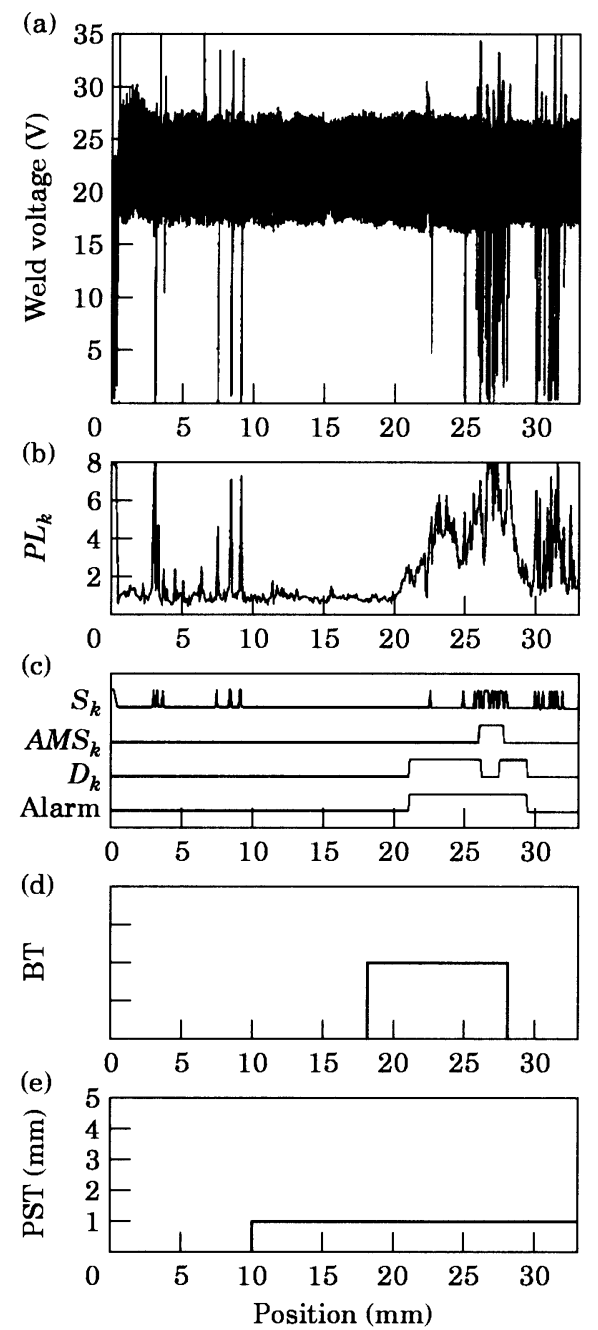

Figure 12. Illustration of the detection of burn-through when welding a T-joint with step disturbance: measured weld voltage is shown in part (a) (Note the different time scale compared with Fig. 6). The corresponding parameters $\mathrm{PL}_{k}$, as well as $\mathrm{S}_{k}, \mathrm{AMS}_{k}, \mathrm{D}_{k}$ and Alarm, are shown in parts (b) and (c) respectively (c.f. with Fig. 11). The actual locus of burn-through (BT) is shown in part (d). The position and type of the step disturbance (PST) are shown in part (e).

Selection of the threshold for the parameter $M P L R_{k}$ is of central importance in the design of the detection algorithm. Two conflicting requirements must be considered. First, the threshold should be low enough to ensure that probability of detection is not too small. Second, the threshold should be high enough to ensure that false alarm probability is not too large. In practice, the false alarm probability must be low when, for example, welding hundreds of meters of a tube. In such a case, the welding process should not be allowed to be stopped every meter due to false alarms. A $10-\mathrm{m}$ weld joint corresponds to approximately $10^{5}$ pulses, yielding a false alarm probability of $P_{F}=10^{-5}$.

The proposed compound detector is designed to detect burn-through in welds. The detector could, however, be used to detect other disturbances in the weld process as well. The process stability, i.e. the characteristics of the welding process should not change in an uncontrolled manner during welding. This is an important factor for ensuring weld 
quality [27]. To the authors' knowledge, a strict definition of process stability does not exist. It seems, however, reasonable that the following criteria must be fulfilled to maintain the stability of a weld process $[5,17,18]$ :

1. Arc stability: background current must exceed a minimum limit for stable arc.

2. Metal transfer: spray type metal transfer must be produced.

3. Arc length: a constant arc length must be maintained.

For example, the short circuitings, which are probably caused by the electrode making contact with the workpiece, indicate that the arc length criterion is not fulfilled in the experiments. Thus, the proposed detector can be used to detect other instabilities in welding. If a disturbance in, for example, wire feed rate or shielding gas flow is reflected in changes in the weld voltage, then the outputs $A M S_{k}$ and $D_{k}$ could be used to trigger a lamp or a bell to alert the welder in charge, indicating that the process is deteriorating. This could give time to correct the process before burn-through, or other weld defects occur.

One specimen for each type of disturbance has been used for developing the detection algorithm, and for setting the alarm limit. The remaining specimens are used to evaluate the proposed detection algorithm. In order to exploit the method fully, further experiments and a larger batch of data should be collected.

\section{REFERENCES}

1. B. ÅGREN 1995 PhD thesis. Lund University, Sweden. Sensor integration for robotic arc welding.

2. P. J. Blakeley 1992 Proceedings of the International Conference on Automated Welding Systems in Manufacturing, Gateshead, U.K., paper 40. Developments in monitoring systems for resistance and arc welding.

3. F. KisSELEVSKI, N. ShVYdKII and V. Dolinenko 1985 Proceedings of the International Conference on Automation and Robotisation in Welding and Allied Processes, Strasbourg, France, 146-150. Mathematical simulation of scanning arc in MIG-welding.

4. J. C. Amson 1965 British Journal of Applied Physics 16, 1169-1179. Lorentz force in the molten tip of an arc electrode.

5. C. J. Allum 1985 British Journal of Applied Physics 7, 1447-1468. Metal transfer in arc welding as a varicose instability: I. Varicose instabilities in a current-carrying liquid cylinder with surface charge.

6. K. Leino, A. NikKola and K. VARTIAINEN 1984 Research report 264, Technical Research Center of Finland, Espoo. Prediction of weld defects using welding condition data.

7. W. LuCAS, N. Ahmed and G. A. Hutt 1983 The Welding Institute Research Bulletin 10, 329-336. Process stability in MIG welding.

8. S. LiU and T. SIEVERT 1989 Welding Journal 2, 52-58. Metal transfer in gas metal arc welding: droplet rate.

9. S. R. Gupta, P. C. Gupta and D. Rehfeldt 1988 Welding Review 11, 232-241. Process stability and spatter generation during dip transfer in MAG welding.

10. B. Ågren, J-O. Gustavsson and S. Adolfsson 1991 Proceedings of EUROJOIN Paris, France, 51-58. Quality monitoring and control for pulsed arc welding of aluminium.

11. S. Adolfsson, K. ERICSON and B. ÅgReN 1994 Research report TULEA 1995:15, Division of Signal Processing, Luleå University of Technology, Sweden. On Automatic Detection of burn-through in GMA welding-weld voltage analysis.

12. S. AdOLFSSON 1995 Research report TULEA 1995:16, Division of Signal Processing, Lulea University of Technology, Sweden. On automatic detection of burn-through using a parametric model.

13. L. LJUNG 1987 System Identification: Theory for the User. Englewood Cliffs, NJ: Prentice Hall.

14. L. LuUng 1991 Sytem Identification Toolbox. The MathWorks, Inc.

15. T. Sö̈ERSTRÖM and P. StolCa 1989 System Identification. Englewood Cliffs, NJ: Prentice Hall.

16. P. L. Connor (ed.) 1987 Welding technology. Volume 1 of Welding Handbook, AWS. Pergamon Press.

17. M. Amin 1981 Metal Construction 6, 349-353, Synergic pulse MIG welding. 
18. M. Amin 1983 Metal Construction 5, 272-278, Pulse current parameters for arc stability and controlled metal transfer in arc welding.

19. K. Goldman 1962 Physics of the Welding Arc, 17-22. Electric arc in argon.

20. C. J. Allum 1983 Metal Construction 6, 347-353. Mig welding-time for a reassessment.

21. J. F. LANCASTER (ed.) 1986 The Physics of Welding. Pergamon Press.

22. G. E. COOK 1983 IEEE Transactions on Industrial Electronics IE-30, Robotic arc welding: Research in sensory feedback control.

23. P. J. Brockwell and R. A. Davis 1991 Time Series: Theory and Methods. Springer-Verlag.

24. M. BASSEVILle and I. V. Nikiforov 1993 Detection of Abrupt Changes: Theory and Application. Prentice-Hall, Inc.

25. A. S. WiLlSKY 1976 Automatica 12, 601-611. A survey of design methods for failure detection in dynamic systems.

26. W. B. Lindgren 1976 Statistical Theory. Macmillan Publishing Co.

27. M. XIE 1992 PhD thesis. Lund University, Sweden. Quality assurance and control for robotic GMA welding.

\section{APPENDIX A: NOMENCLATURE}

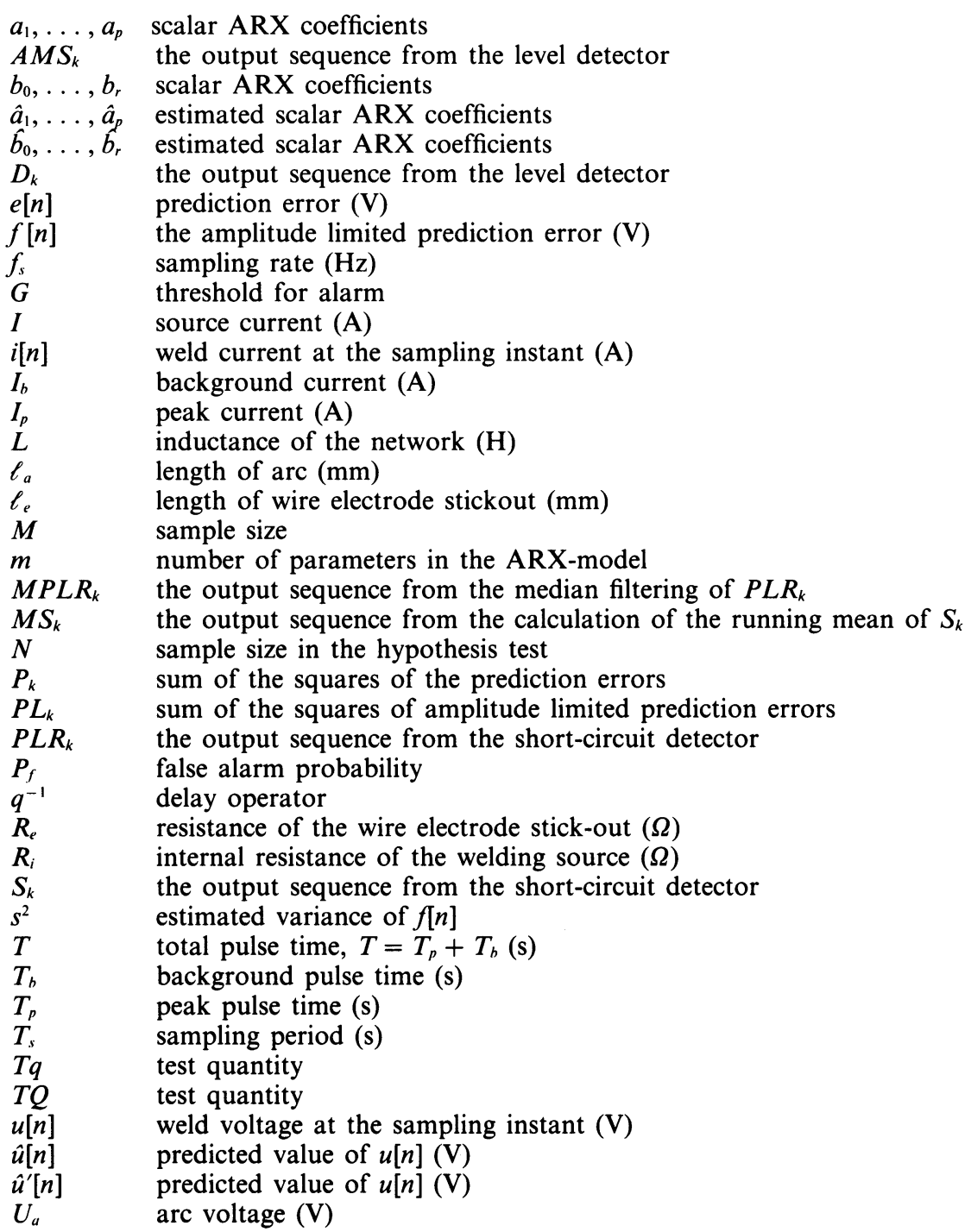




$\begin{array}{ll}U_{a n} & \text { anode voltage fall }(\mathrm{V}) \\ U_{b} & \text { background voltage }(\mathrm{V}) \\ U_{c} & \text { cathode voltage fall (V) } \\ U_{c o} & \text { column voltage fall }(\mathrm{V}) \\ U_{e} & \text { wire electrode stick-out voltage }(\mathrm{V}) \\ U_{p} & \text { peak voltage }(\mathrm{V}) \\ U_{w} & \text { weld voltage, } U_{\mathrm{w}}=U_{e}+U_{a}(\mathrm{~V}) \\ w[n] & \text { the signal after the prediction filter }(\mathrm{V}) \\ W_{b} & \text { wire burn-off rate }(\mathrm{mm} / \mathrm{s}) \\ W_{f} & \text { wire feed rate }(\mathrm{mm} / \mathrm{s}) \\ \varepsilon[n] & \text { white noise process of the ARX-process } \\ \sigma^{2} & \text { variance of } \varepsilon[n] \\ \hat{\sigma}^{2} & \text { estimated variance of } \varepsilon[n] \\ \chi_{N}^{2} & \text { chi-squared with } N \text { degrees of freedom }\end{array}$

